\title{
Andrei Zelevinsky
}

Published online: 27 November 2013

(C) Springer Science+Business Media New York 2013

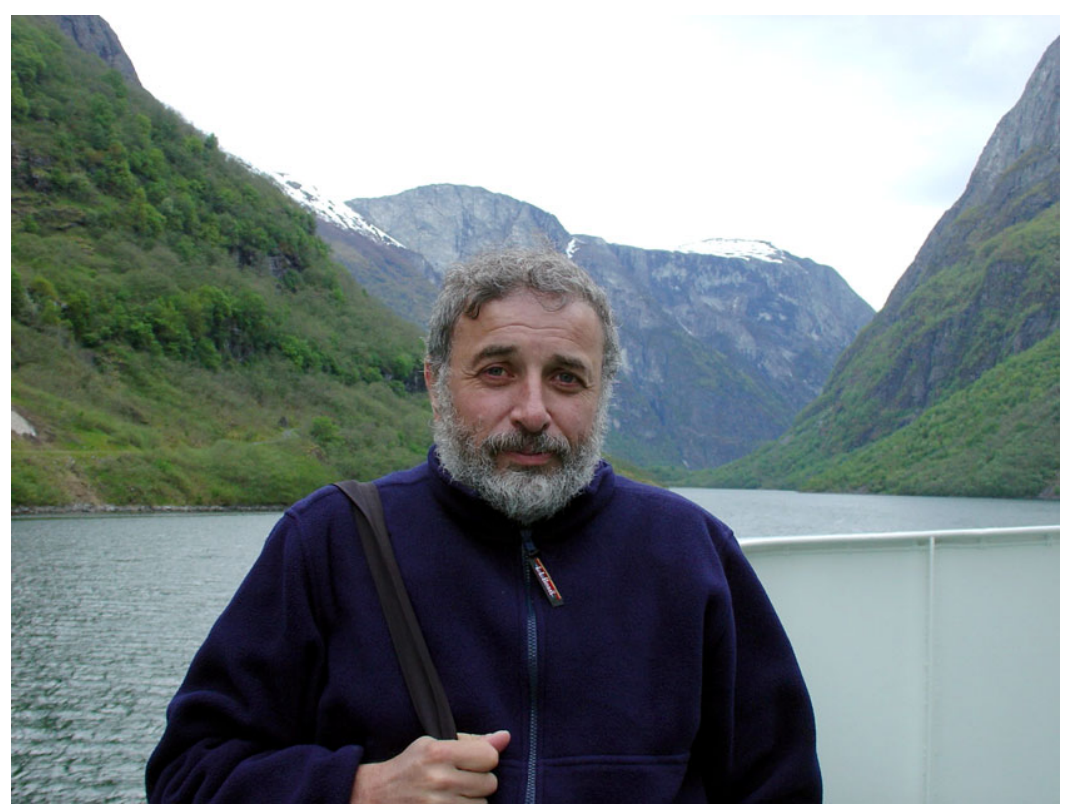

Photo courtesy of Karen Zelevinsky

Andrei Zelevinsky died in April 2013, only a few months after he turned sixty. He served as a member of the editorial board of the Journal of Algebraic Combinatorics since the first issue of the journal was published in 1992. The very first paper of the first issue of the journal was authored by him (jointly with Berenstein). Zelevinsky made major contributions to several areas of mathematics, including representation theory, algebraic geometry, algebraic and polyhedral combinatorics. Among his many fundamental discoveries in algebraic combinatorics, our readers are no doubt familiar with the Gelfand-Kapranov-Zelevinsky theory of generalized hypergeometric functions and Fomin-Zelevinsky's cluster algebras.

Although his name will permanently disappear from the editorial board after this issue, we are certain that his beautiful mathematical theories, ideas, and insights will continue to influence and inspire our authors and readers for many years to come.

Christos A. Athanasiadis, Jonathan Brundan, Thomas Lam, Akihiro Munemasa, Isabella Novik, Hendrik Van Maldeghem 